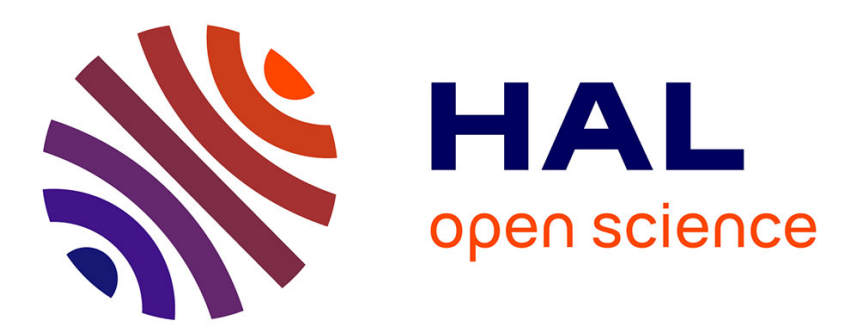

\title{
Virtual and unified address assignment for continuous communication in mobile networks
}

\author{
Robson Melo, Farouk Mezghani, Michele Nogueira, Aldri Santos
}

\section{To cite this version:}

Robson Melo, Farouk Mezghani, Michele Nogueira, Aldri Santos. Virtual and unified address assignment for continuous communication in mobile networks. IFIP Wireless Days (WD 2016), Mar 2016, Toulouse, France. pp.1-6, 10.1109/WD.2016.7461518 . hal-03159049

\section{HAL Id: hal-03159049 \\ https://hal.science/hal-03159049}

Submitted on 9 Mar 2021

HAL is a multi-disciplinary open access archive for the deposit and dissemination of scientific research documents, whether they are published or not. The documents may come from teaching and research institutions in France or abroad, or from public or private research centers.
L'archive ouverte pluridisciplinaire HAL, est destinée au dépôt et à la diffusion de documents scientifiques de niveau recherche, publiés ou non, émanant des établissements d'enseignement et de recherche français ou étrangers, des laboratoires publics ou privés. 


\title{
Virtual and Unified Address Assignment for Continuous Communication in Mobile Networks
}

\author{
Robson Melo*, Farouk Mezghani ${ }^{\dagger}$, Michele Nogueira*, Aldri Santos* \\ *Federal University of Paraná - UFPR, Curitiba, Brazil \\ †Université de Toulouse, INP/ENSEEIHT, IRIT, Toulouse, France \\ * $\{$ rgmelo, michele, aldri $\} @$ inf.ufpr.br, ${ }^{\dagger}$ farouk.mezghani@enseeiht.fr
}

\begin{abstract}
Mobile devices connections to heterogeneous networks require the management of the network interfaces, as well as addressing service for each access network. However, the frequent association and dissociation of devices to the network and several communications patterns hamper the address control and negotiation. In general, addressing strategies for heterogeneous wireless networks are individualized and do not adapt fast enough to several address patterns from each communication technology, hindering the seamless handoffs. This paper presents an innovative dynamic address scheme for heterogeneous wireless networks founded on Network Function Virtualization (NFV) approach aiming to support the avoidance of interruptions in end-to-end communications and providing seamless handoffs. It is described a method for the unification of the network address formats on mobile devices and for the negotiation of dynamic addresses in order to offer continuous communication. The proposed method has been evaluated by simulations, taking into account WiFi and WiMAX communication technologies. Simulation results show the strategy effectiveness and the maintenance of the end-to-end communication under handoff scenarios.
\end{abstract}

Index Terms-Continuous Connectivity, Dynamic addressing, Heterogeneous Networks, Network Function Virtualization

\section{INTRODUCTION}

A range of new services offered by communication networks has influenced the interaction among people in society. The main change lies in the diversification of services provided though portable devices, yielding a huge demand for interrupt communication during their mobility in heterogeneous wireless networks [1]. The vertical handoff of mobile devices (nodes) requires the effective management of network interfaces and a method to negotiate dynamic addresses for each access network in which the devices are associated. Mobile devices must adjust their addresses according to standards of each network [2]. Thus, supporting the assignment of mobile devices addresses becomes a challenging task in terms of heterogeneous and overlapped wireless networks [3].

Different strategies to manage the address assignment service on mobile devices have been recently proposed for heterogeneous networks. Those strategies are founded on Service Level Agreements (SLAs) [4], ontologies [5], cryptographic keys [6] and other techniques. Also, some works present comparative studies of address assignment schemes for mobile ad hoc networks under different categories and classifications [7], [8], [9]. In [10], the authors propose an address attribution scheme starting from devices previously registered in the network. Although their advances and contributions, the previously proposed schemes are not enough to satisfy the con- tinuous communication requirement during vertical handoffs due to the necessity of reconfiguration to network interfaces. Although these solutions handle of the addresses management, they are still specific for each network technology.

This work presents a dynamic address assignment scheme founded on Network Functions Virtualization (NFV) approach [11]. Its main goal lies in maintaining continuous communication for mobile devices during vertical handoffs, i.e., when mobile devices dissociate from a network and associate themselves to another that employs a different communication technology. In general, vertical handoffs interrupt the communication since network interface address needs to be reconfigured and adapted in accordance with the technology standard the node will follow. Hence, the proposed address assignment scheme is self-managing and based on an unified and virtual address format to avoid data flow interruption. This scheme supports the smooth transition encapsulating different address formats in a unified model.

An evaluation of the proposed scheme considering different scenarios shows that the process of dynamic addresses negotiation is effective over vertical handoffs. The analysis of negotiation time, delivery, rate, and latency assesses the impact caused in the dynamic address assignment on the communications of heterogeneous access networks. Results show that the overlying networks density and the velocity of the mobile device have influence on the addresses negotiations.

This paper proceeds as follows. Section II presents related work. Section III describes the dynamic addressing system. Section IV describes the performance evaluation of the proposed method. Finally, Section V concludes the paper.

\section{RELATED WORK}

Managing address assignment in dynamic and heterogeneous wireless networks is a demanding task. Studies, such as [9], [8], [7], have compared address assignment schemes for mobile ad hoc networks, based on different characteristics to compare dynamic and automated self-configuration. Maurizio et al. [4] discussed address assignment in heterogeneous networks by Service Level Agreements (SLAs) to automate devices configuration. In [5], authors examined the application of ontologies in address assignment management on heterogeneous multi-layer network. The authors goal was on managing topologies using Network Management Systems (NMS) to automatically discover all the devices within the network coverage area and report their properties. However, 
despite the innovation in the use of ontologies, the proposal is not enough to support continuous communication.

Bengt et al. [6] presented an architecture to identify interconnected nodes and devices using cryptographic keys to assure connectivity in heterogeneous wireless networks. However, they do not present a method for negotiating dynamic addresses on vertical handoffs. In [2], the authors present an addressing proposal for overlapping coverage areas. That proposal creates an overlay network over different communication technologies to allow communication to mobile devices and route messages. The overlay network provides homogeneous and persistent connectivity despite the heterogeneity of the underlying networks and devices mobility. Although the strategy is applied in heterogeneous networks, the work focuses on P2P communication, and the address negotiation model for devices entering the network is not handled.

Mazen et al. [10] proposed a dynamic IP address assignment architecture for wireless sensors networks. The phase of address assignments tries to assure a communication channel between previously registered devices. This work is focused on IP addresses, which not always can be used in the context of heterogeneous wireless networks, such as cellular networks. Another issue uncovered lies in the communication maintenance during vertical handoffs. Uttam et al. [12] examined the difficulty in securing and distributing IP addresses uniquely in MANETs. They proposed an address allocation algorithm based on a dynamic IP configuration distribution scheme for hosts authorized to transmit data in the network. Each host can generate unique IP addresses from its own IP address and can attribute these addresses to new devices entering the network. The distribution of addresses is inspired by the method of distributing cryptographic keys. The two studies also present a signature scheme to prove authentication during transmission of the address allocation message. Despite touching security, their focus lies on the distribution of unique addresses.

The current proposals are not still enough to guarantee continuous communication under vertical handoffs, since the address formats are not uniform and suitable for different access networks. Hence, it is needed robust solutions that support the continuous communication on several technologies.

\section{UNified Virtual AdDRESS ASSignMENT SCHEME FOR HETEROGENEOUS WIRELESS NETWORKS}

This section presents an address assignment scheme based on the Network Function Virtualization (NFV) approach. It employs a virtual and unified address, reducing the need of human intervention in the management process. The scheme encompasses two steps: the first consists in a method to unify address formats, and the second defines the addresses assigning in heterogeneous networks.

\section{A. Method for unifying the address formats}

Unifying the address format allows devices to connect to different communication technologies without interruption on the data flow. The unification of the address formats consists in dividing the address into two parts, as showed in Fig. 1. One part corresponds to the Network identifier - NetID (regardless of technology) and the other to the Device identifier - HostID.

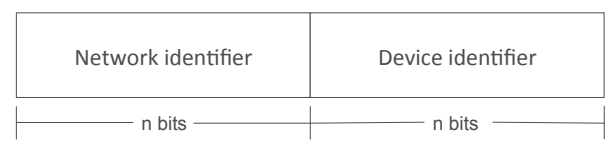

Fig. 1. Strategy for unification of the address formats

NetID consists in a chain of 48 bits with a single value, known as BSSID (Basic Service Set Identification). The BSSID corresponds to the MAC (Media Access Control) address of the access provider. This approach assures that each network will be uniquely identified, enabling the senders to identify the data packets destinations. HostID consists in another chain of 32 bits defined by IP format, responsible for identifying the device within the network. A hash function is used to determine device identifiers of 32 bits in network technology that have higher values. HostID enables conformation of various address formats to a single standard, independent of the communication technology.

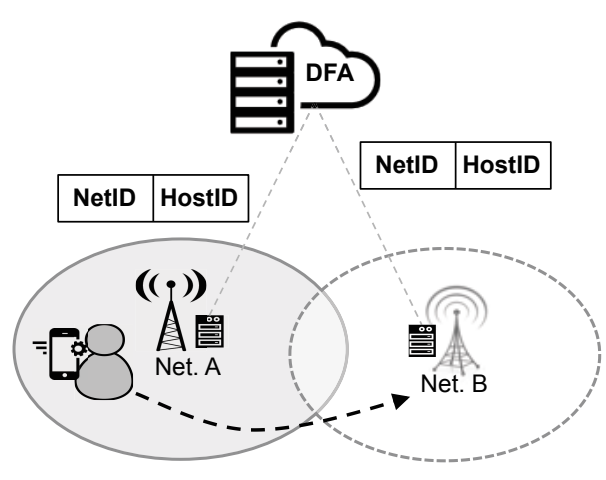

Fig. 2. Operation of the addressing scheme

The address allocation of mobile devices by requesting access to the network demands an appropriate addressing format for seamless vertical handoff. The virtualizated addresses allocation and distribution process allows handoffs without the adaptation of network addresses, since this process takes place virtually independent of the type technology communication, as showed in Fig 2. The virtualized addresses negotiation requires a second step to maintain an uninterrupted communication over handoffs, and this step is detailed below.

\section{B. Address negotiation method}

This method is defined by a DFA (deterministic finite automaton), because it allows us to represent a simplified way the events and state transitions of a system. The DFA consists of a 5 -tuple $\left(Q, \sum, \delta, q_{0}, F\right)$ where:

- $Q$ corresponds to a finite set of states;

- $\sum$ consists in a finite set of input symbols called Alphabet;

- $\delta: Q \times \sum \rightarrow Q$ comprises a transition function;

- $q_{0} \in Q$ indicates an initial state;

- $F \subseteq Q$ represents a set of accepting states. 
Let $w=a_{1}, a_{2}, \ldots, a_{n}$ a string of symbols over the alphabet $\delta$, the automaton accepts a string $w$ if and only if there is a sequence of states, $r_{0}, r_{1}, \ldots, r_{n}$ in $Q$ with the conditions:

$$
\begin{array}{r}
r_{0}=q_{0} \\
r_{i+1}=\delta\left(r_{i}, a_{i+1}\right), \text { for } i=0, \ldots, n-1 \\
r_{n} \in F
\end{array}
$$

The first condition states that the automaton $M$, illustrated in Fig. 3, starts in the initial state $q_{0}$. The second is that, since each symbol of the input $w$, the automaton moves from state to state according to the transition function $\delta$. The third and last condition says that the automaton accepts $w$ if and only if the last symbol of the input leads to the automaton stops in a final state $f$, such that $f \in F$. Otherwise, the automaton rejects the input. The set of strings accepted that $M$ is called Language recognized by $M$ and is symbolically represented by $L(M)$. The DFA $M$, which is the process of trading address is formally defined as follows:

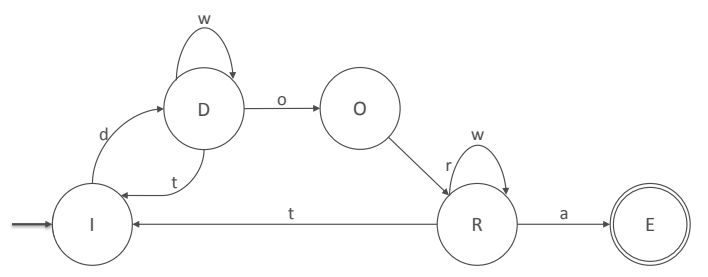

Fig. 3. Deterministic finite automaton $M$

- A finite set of states $Q=\{I, D, O, R, E\}$ respectively representing the set of machine states $M=$ \{NetworkInput, ServerDiscovery, OfferReception, AddressRequest, AddressAttributed \};

- The alphabet $\sum=\{d, o, r, a, w, t\}$ representing the events occurring in the system, $\{$ SendServerDiscover, ReceivesLeaseOffer, SendAddressRequest, ReceivesAddressACK, WaitsResponse, TimeOut $\}$;

- The transition function $\left(\delta: Q \times \sum \rightarrow Q\right)$, which includes the acceptance of the conditions in which events happen. Table I show all transition functions of DFA.

TABLE I

TABLE TRANSITION STATES OF $M$

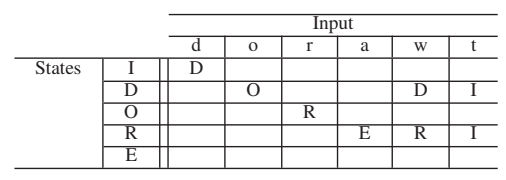

- The initial state $q_{0}=I$, such that $\left(q_{0} \in Q\right)$, representing the state NetworkInput.

- The set of accepting states $F=E$, such that $(F \subseteq Q)$ representing the final state AddressAttributed.

When a device sends an access request to connect to a network, an address must be assigned to it, so that its data transmission can start and continue. Hence, the device needs to discover address servers on the network. Fig. 4 illustrates the procedure where the device (client) in transition by different networks starts a negotiation process broadcasting a server discovery message, including its HostID. When the address server receives this message, it calculates the address to be assigned, i. e., the NetID and HostID values. The calculation of address is made by joining the chain of 48 bits in NetID and 32 bits in HostID. Next, this server stores the address locally and sends an acceptance offer message to the device. Upon receiving the acceptance offer, the client then sends an address request message to the detected server. Finally, the server updates its list of assigned addresses and sends an address acknowledgment address ACK message, confirming the address assigned to the client. At this moment, with the attributed address, the client can start or continue data transmissions via the new access network.

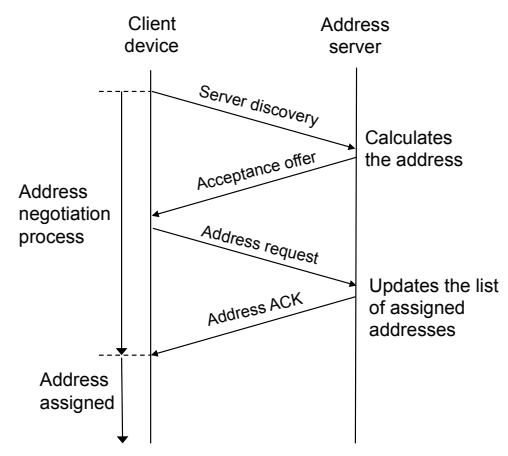

Fig. 4. Address negotiation process

\section{Performance Evaluation}

Simulations were employed to validate the address assignment scheme proposed for heterogeneous network. The scheme was implemented and added as a module of the Network Simulator 2 (NS-2), version 29, since that it allows operational testings of the scheme on different types of networks. An extension of the Agent class on NS-2 has been implemented to enable the exchange of messages on the negotiation process. Also, the Packet class has been changed to allow the exchange of new control messages for supporting the virtual address assignment. We have applied the Handoff class, implemented by NIST [13], [14], to control the handoff process and start the address negotiation process.

Three scenarios have been evaluated considering factors as the node maximum speed and the overlapping networks density. The levels for the maximum speed factor are $1 \mathrm{~m} / \mathrm{s}$ representing a pedestrian, $3 \mathrm{~m} / \mathrm{s}$ representing a cyclist and a $12 \mathrm{~m} / \mathrm{s}$ representing a car in an urban area. The random way point mobility model has been employed. The overlapping network density factor has as levels: a domestic area as a home with a Low Overlapping Network (LON) density (two overlapping wireless networks), a residential area as a neighborhood or a set of apartments in a building with Average Overlapping Network (AON) density (ten overlapping wireless networks), and a shopping mall area with High Overlapping Network density (HON) (twenty overlapping wireless networks). All devices offer WiFi or WiMAX communication technologies, 
but other technologies could have been applied. Fig. 5 shows scenarios, and Table II shows parameters and values applied.

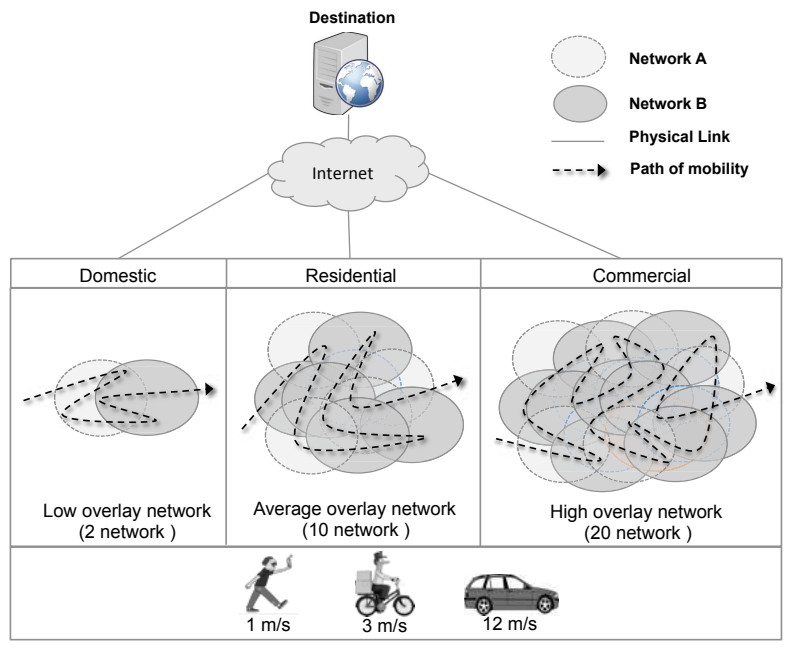

Fig. 5. Evaluation Scenarios

TABLE II

PARAMETERS OF THE SIMULATION SYSTEM

\begin{tabular}{|l|c|c|}
\hline \multicolumn{1}{|c|}{ Parameter } & \multicolumn{2}{|c|}{ Value } \\
\hline \multirow{2}{*}{ Areas } & Domestic & $100 \mathrm{~m} \times 100 \mathrm{~m}$ \\
\cline { 2 - 3 } & Residential & $150 \mathrm{~m} \times 150 \mathrm{~m}$ \\
\cline { 2 - 3 } & Commercial & $200 \mathrm{~m} \times 200 \mathrm{~m}$ \\
\hline \multirow{3}{*}{ Overlay Networks } & Low & 2 \\
\cline { 2 - 3 } & Average & 10 \\
\cline { 2 - 3 } & High & 20 \\
\hline Coverage radius & \multicolumn{2}{|c|}{$100 \mathrm{~m}$} \\
\hline Mobile Interfaces & \multicolumn{2}{|c|}{ WiFi and WiMAX } \\
\hline Velocity mobility & \multicolumn{2}{|c|}{$1 \mathrm{~m} / 3 \mathrm{~m} / \mathrm{s} ; 12 \mathrm{~m} / \mathrm{s}$} \\
\hline Simulation time & \multicolumn{2}{|c|}{$200 \mathrm{~s}$} \\
\hline Bandwidth & \multicolumn{2}{|c|}{$10 \mathrm{Mbp} / \mathrm{s}$} \\
\hline Application & \multicolumn{2}{|c|}{ CBR over UDP } \\
\hline
\end{tabular}

The evaluation measures three metrics: Number of addresses negotiated - checks the effectiveness of address negotiations during vertical handoffs; Delivery rate - means the amount of delivered messages to destination; Latency - indicates the delay caused by the scheme in the network data flow.

\section{A. Results}

Fig. 6 shows the number of address negotiations over the three levels for maximum velocity (groups of bars) and the three overlapping network densities (each represented by a bar color). Over $L O N$ density, the address negotiation process presents a similar behavior regardless of the devices velocity. This behavior occurs because there are only two overlapping networks on LON, and no matter the variation in velocity, the devices can only transit from one network to the other.

Over $A O N$, the number of negotiations increases, depending on the device's velocity. This happens because each mobile device detects a higher number of available networks in its coverage area, thus it makes faster dissociations/associations on the networks. Over HON, when devices travel in low velocity (pedestrian), even when there are many overlapping networks, the number of negotiations is low. The reason is that the device is not traveling fast enough to pass through many coverage areas. Hence, under low velocity, the device

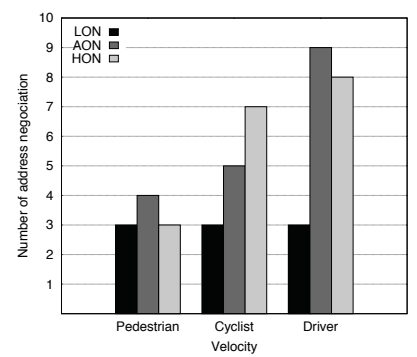

Fig. 6. Total of address negotiations

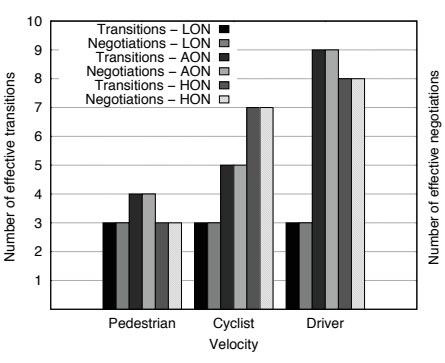

Fig. 7. Effectiveness - address negotiations has low mobility and carries out only few negotiations. For the bicycle rider, the number of networks through which the device passes increases, then the number of address negotiations also rises considerably. Over high velocity (car), results show that despite the high mobility, devices carry out fewer negotiations than if they are in an area with medium overlapping density.

The effectiveness of the virtual address assignment scheme is assessed by the ratio of the number of addresses negotiations and the number of transitions performed by the mobile device in different access networks. Results point out to equality between the number of address negotiations and number of transitions by mobile devices. This result confirms that the negotiation process occurs for each device handoff, Fig. 7 shows the number of effective transitions over all evaluated scenarios.

Fig. 8 shows the network conditions before, during and after the address negotiation in terms of the delivery ratio. This metric intends to check the impact of the address negotiation process over the network. The handoff moment, and hence the moment of address negotiation is represented by the dotted lines. Over $L O N$ and $A O N$ densities, the yielded delivery ratios are very similar (Fig. 8(a) and $8(\mathrm{~b})$ ). The variations before and after the negotiation process are similar. This result is observed regardless the mobile device velocity. In Fig. 8(c), the variation on delivery ratio is greater after the negotiation process, which can be explained by the characteristics of the network to which the device migrates. This behavior shows that the variation of the delivery rate is influenced by the kind of network to which the device is connected, and not by the address negotiation process.

Over $A O N$ density (Fig. 9), variations in the delivery ratio occur near to the address negotiation processes. However, this process does not influence the delivery ratio. For pedestrian mobility (Fig. 9(a)), the delivery rate oscillates according to the conditions of the networks. As the device migrates to a more stable network, its delivery rate value also stabilizes. For the velocity of a cyclist (Fig. 9(b)), the delivery rate remains constant independent of the number of transitions, with only a few moments of variations. Finally, for devices with high mobility (Fig. 9(c)), there are highest variations of the delivery rate. This occurs because high mobility influences the number of transitions to different networks and each network can have different operating conditions and characteristics, affecting the delivery rate values. 


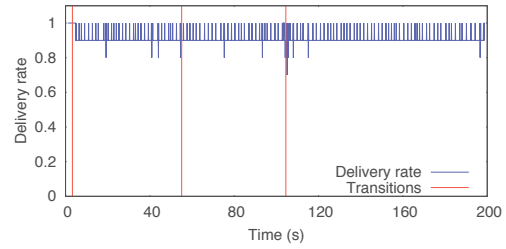

(a) Pedestrian

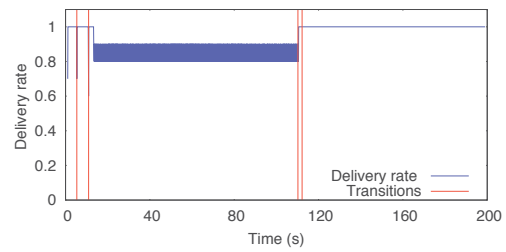

(a) Pedestrian

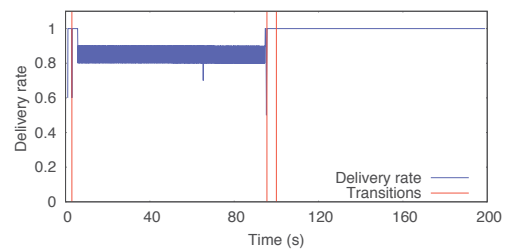

(a) Pedestrian

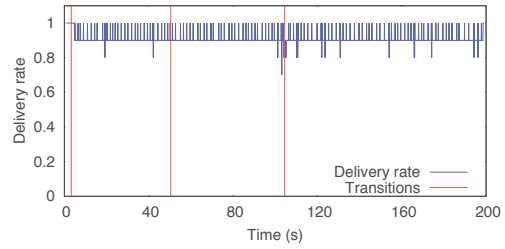

(b) Cyclist

Fig. 8. Delivery rate in LON scenarios

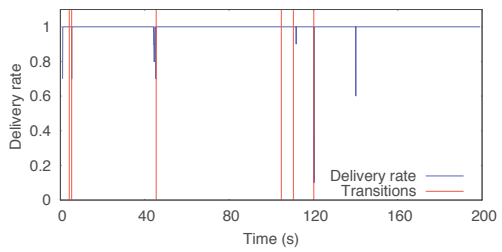

(b) Cyclist

Fig. 9. Delivery rate in AON scenarios

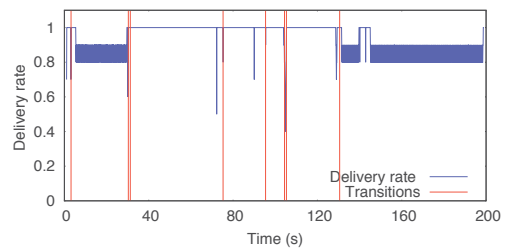

(b) Cyclist

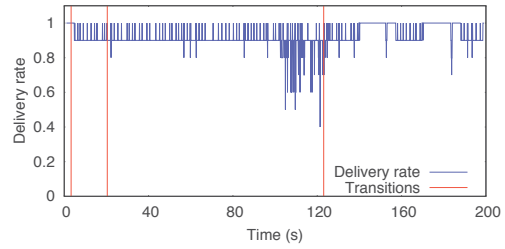

(c) Driver

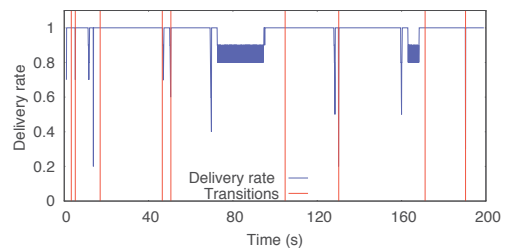

(c) Driver

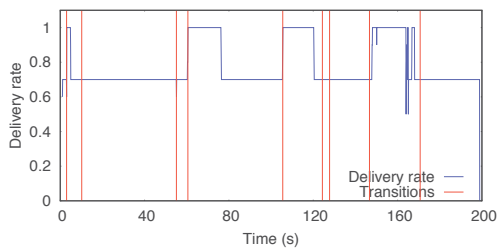

(c) Driver

Fig. 10. Delivery rate in HON scenarios

Over HON density, Fig. 10 shows that faster is the velocity, more negotiations occur. Then, the delivery ratio presents a greater variation. On pedestrian velocity (Fig. 10(a)) there is a certain stability in the delivery ratio, which is affected during the migration of the connection from one network to another. On the bicycle velocity (Fig. 10(b)), the same behavior occurs, but there are greater variations due to the increase in the number of transitions caused by the higher velocity on high network overlapping density. On car velocity (Fig. 10(c)), the delivery rate is highest in the $H O N$ scenario. The faster velocity of the device means it makes more transitions.

Comparison of the delivery rate and address negotiation duration shows that the reduction in the delivery ratio is not directly related to the negotiation process. It is due to the characteristics of the network to which the device connects. This occurs due to the greater exchange of information between the device and the network in reason of events, such as link detected, link up and link down events, which are carried out as new networks are detected in the same coverage area. These events start the process of assessing the conditions of the network to enable transition of the access to the best available network. Results show the address negotiation process is only influenced by the network characteristics.

Latency was assessed to measure the efficiency and impact of the negotiation process on the data flows. Over LON density (Fig. 11), the achieved latency had small variations after the transition from one network to another. On pedestrian velocity (Fig. 11(a)) and cyclist velocity (Fig. 11(b)), latency remained at approximately 0.5 second. The behavior is almost the same due to the similarities in velocities, causing a small impact on the network. On car velocity (Fig. 11(c)), the latency varies due to the transition and connection maintenance to a network with different conditions, such as greater throughput.

Over $A O N$ density (Fig. 12), the latency also varies in relation to the conditions of the new network. Fig. 12(a) shows the case of a device operated by a pedestrian, where the results vary according to the migration of the connection to a network with different technology. However, in moments when the migration is between networks with the same technology, the latency value remains stable, as shown in Fig. 12(b), in which a cyclist passes from one network to another with the same technology. On high mobility (Fig. 12(c)), the device's velocity has a strong influence on the variation of the results, which oscillate regardless of the number or transitions carried out.

Over HON density, the node latency is affected by the node transition between networks, where the network's technology determines its operating characteristics and conditions. On pedestrian velocity (Fig. 13(a)), the latency varies as the device migrates to a network with different technology. Due to the low mobility and longer period in the same network after migration, the number of address negotiations diminishes, as the variation in latency. Fig. 13(b) shows similar behavior than Fig. 13(a). However, due to the higher mobility, the device performs more transitions and its latency variation is greater. On high mobility (Fig. 13(c)), the transitions between networks is higher, resulting in variations over latency. 


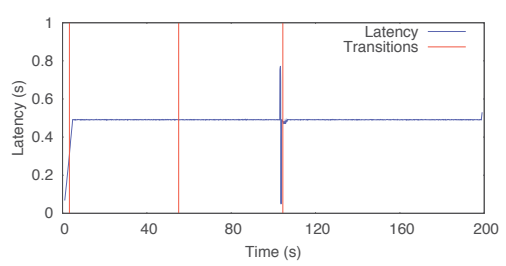

(a) Pedestrian

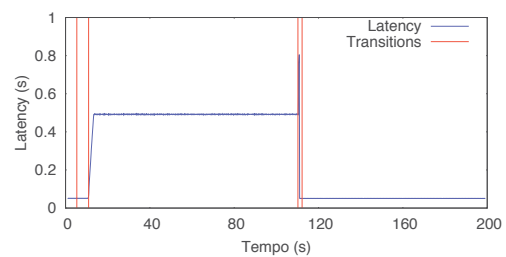

(a) Pedestrian

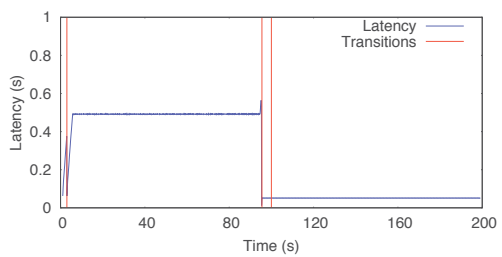

(a) Pedestrian

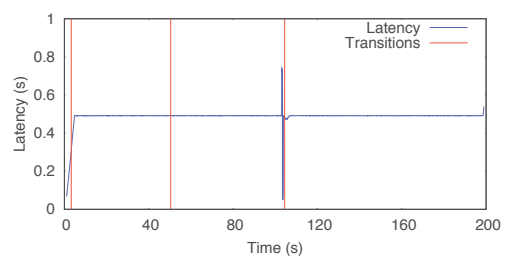

(b) Cyclist

Fig. 11. Latency in LON scenarios

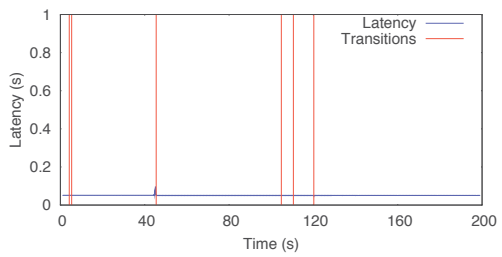

(b) Cyclist

Fig. 12. Latency in AON scenarios

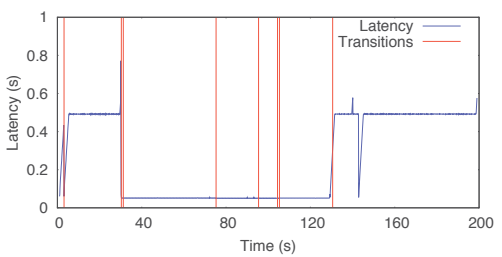

(b) Cyclist

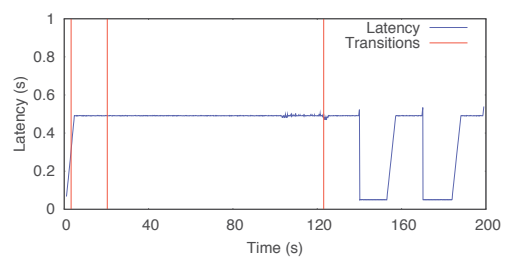

(c) Driver

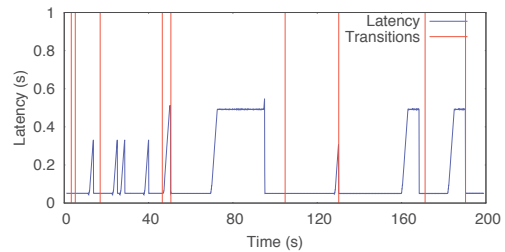

(c) Driver

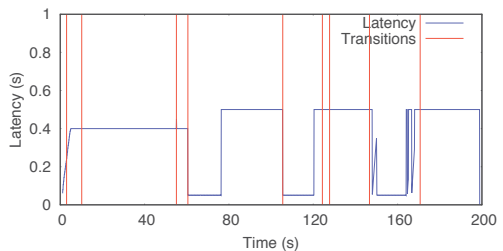

(c) Driver

Fig. 13. Latency in HON scenarios

The results of assessing the addressing system's efficiency show that the address negotiation process does not have a significant impact on the data flow in networks. The delivery rate and latency are not influenced by execution of the addressing system. Finally, the results indicate that these metrics are affected only by the characteristics of each network instead of by the process of adapting the address of devices in transition between heterogeneous access networks.

\section{CONCLUSION}

This paper presented a dynamic and virtualized address scheme for heterogeneous wireless networks in order to support the prevention of interruptions in end-to-end communications by providing seamless handoffs. The continuous communication of mobile devices is achieved by scheme due to a method for the unification of the network address formats on mobile devices and for the negotiation of dynamic addresses. Simulation results considering different scenarios with Wi-Fi and WiMAX standards and coverage areas have showed the effectiveness of scheme in terms of number of negotiation addresses, delivery ratio and latency. As future work, we aim to apply this scheme to support the development of other services, such as authentication and reliable data exchange, ensuring continuous connectivity of devices in handoff over heterogeneous wireless networks.

\section{REFERENCES}

[1] S. Chen, Y. Shi, B. Hu, and M. Ai, "Mobility-driven networks (mdn): from evolution to visions of mobility management," IEEE Network, vol. 28 , no. 4 , pp. 66-73, 2014.
[2] O. Waldhorst, "On overlay-based addressing and routing in heterogeneous future networks," in IEEE ICCCN, 2010, pp. 1-8.

3] V. C. M. Borges, K. V. Cardoso, E. Cerqueira, M. Nogueira, and A. Santos, "Aspirations, challenges, and open issues for software-based $5 \mathrm{~g}$ networks in extremely dense and heterogeneous scenarios," Wireless Communications and Networking, 2015.

[4] M. D'Arienzo, A. Pescapè, and G. Ventre, "Dynamic service management in heterogeneous networks," Journal of Network and Systems Management, vol. 12, no. 3, 2004

[5] L. Frye and L. Cheng, "A network management system for a heterogeneous, multi-tier network," in IEEE GLOBECOM 2010, 2010, pp. 1-5

[6] B. Ahlgren, J. Arkko, L. Eggert, and J. Rajahalme, "A node identity internetworking architecture," in IEEE INFOCOM, 2006, pp. 1-6.

[7] R. Rohit and D. Singh, "A study of various address allocation schemes for mobile ad hoc networks," IJETTCS, 2014.

[8] V. Hemamalini and G. D. Zayaraz, "A survey on ip configuration of mobile ad hoc networks with and without dad mechanism," nternational Journal of Scientific and Research Publications, 2013.

[9] Y. Sun and E. M. Belding-royer, "Dynamic address configuration in mobile ad hoc networks," University of California, Santa Barbara, Department of Computer Science, Tech. Rep., 2003.

[10] M. Khair, B. Kantarci, and H. Mouftah, "Towards cellular ip address assignment in wireless heterogeneous sensor networks," in IEEE ISCC, 2011, pp. 615-619.

[11] N. F. I. S. Group, "Network functions virtualisation (nfv): Network operator perspectives on industry progress," in SDN and OpenFlow World Congres. European Telecommunications Standards Institute (ETSI), Frankfurt-Germany, 2013.

[12] U. Ghosh and R. Datta, "A novel signature scheme to secure distributed dynamic address configuration protocol in mobile ad hoc networks," in IEEE WCNC, 2012.

[13] H. Marques, J. Ribeiro, P. Marques, and J. Rodriguez, "Simulation of 802.21 handovers using ns-2," Journal of Computer Systems, Networks, and Communications technologies, architectures, and services, 2010.

[14] NIST, "The network simulator ns-2. nist add-on. ieee 802.21 model," National Institute of Standards and Technology (NIST), Tech. Rep., 2006. 